\section{Copernicus User Uptake - Copernicus Relays and the Copernicus Academy}

Gl_Forum 2017, Issue 1

Page: 253 - 259

Short Paper

Corresponding Author:

zeil@spatial-services.com

DOI: 10.1553/giscience2017_01_s253

\author{
Peter Zeil', Stephane Ourevitch², Annekatrien Debien² and Udrivolf Pico² \\ 'Spatial Services GmbH, Salzburg, Austria \\ 2SpaceTec Partners SPRL, Brussels, Belgium
}

\begin{abstract}
The Copernicus programme is expected to provide important strategic, social and economic benefits to the European Union. In order to ensure that the programme delivers its benefits, it is essential to deploy an effective user engagement strategy for stimulating the uptake of Copernicus data, services and information. The approach adopted by the European Commission is based on three core principles: (a) public authorities have a central role to play; (b) a stronger implication of the commercial downstream sector is needed; and (c) the European Commission should better leverage existing structures. Key elements of the user uptake concept are the Copernicus Relays and the Copernicus Academy, both supported by the Copernicus Support Office. This paper serves to raise awareness of User Uptake activities and to inform about the status of its implementation.
\end{abstract}

\title{
Keywords:
}

Copernicus, user uptake, Copernicus Relays, Copernicus Academy

\section{The context}

The Copernicus programme is expected to provide important strategic, social and economic benefits to the European Union. In order to ensure that the programme delivers its benefits according to the set expectations, it is essential to deploy an effective user engagement strategy for stimulating the uptake of Copernicus data, services and information. Numerous user uptake initiatives have been undertaken over recent years or are currently under development, many of which are supported by the European Commission. Past experience has shown, however, that there is a clear need for a systemic and integrated framework to ensure the continuity and sustainability of these initiatives. Furthermore, the diversity of the (potential) user communities in the public and private sectors and the dispersion of users at different geographic levels within public authorities mean that user uptake initiatives are complex to manage. 
In the framework of Copernicus user uptake activities, the Commission has undertaken a study with the purpose of identifying strengths and gaps in existing user and market uptake initiatives across the Copernicus Participating Countries (EC, 2016a).

Whilst the study identified many positive uptake activities, it also highlighted a need for coordination between these various initiatives. An important recommendation was to build an extended Copernicus User Uptake Network to enlarge the outreach into the various Copernicus Participating Countries, and to leverage the user uptake initiatives across borders. To build this Network, it was paramount to establish a Copernicus User Uptake Support Office, the role of which is to coordinate and support the development and implementation of the Copernicus User Uptake Core Tools, a toolbox aiming to stimulate user uptake.

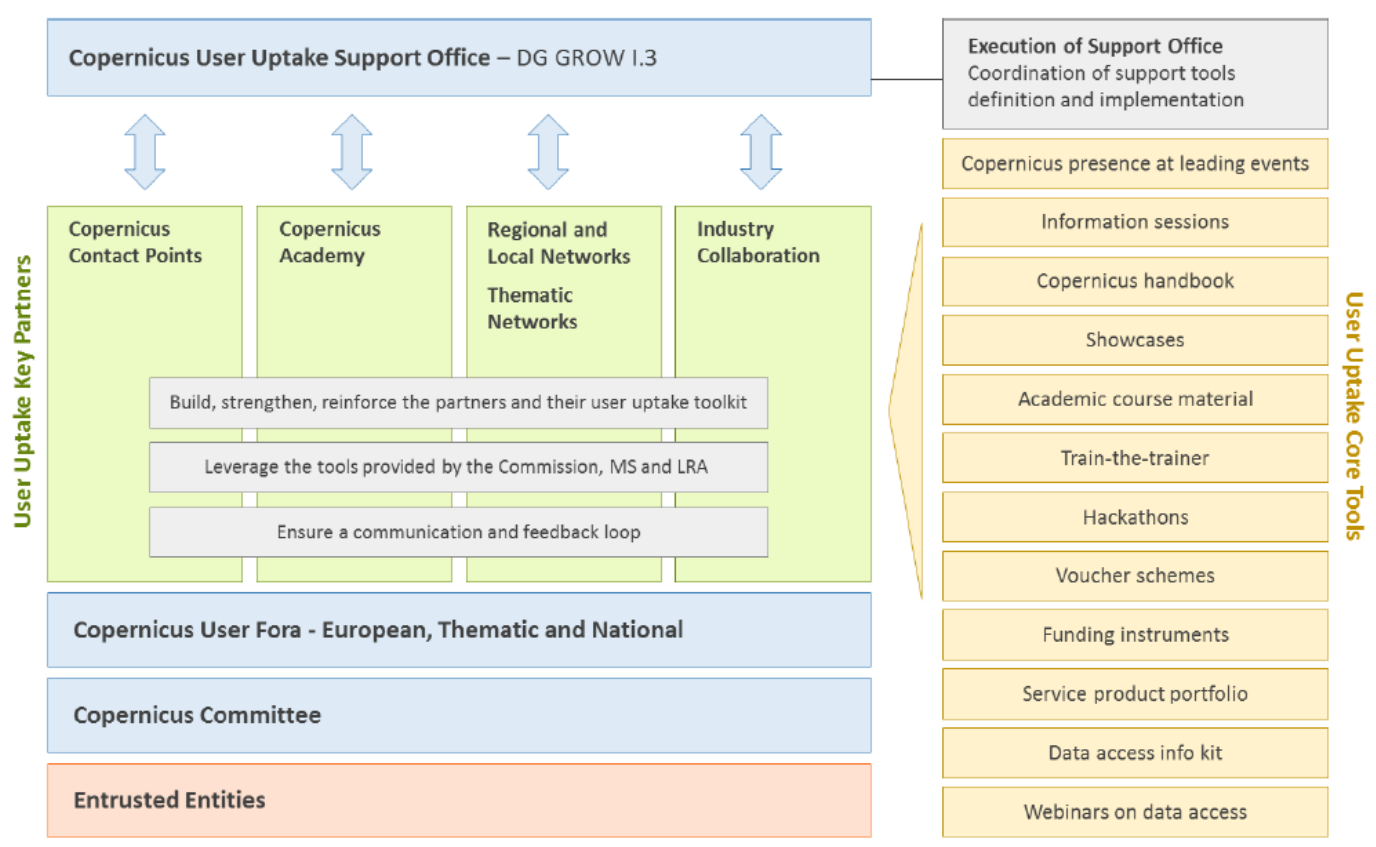

Figure 1: Copernicus User Uptake Network Architecture (EC,2016a)

The Commission therefore decided to develop different channels of promotion, targeting intermediate and end-user communities. An awareness structure is to be set-up to ensure a mid- to long-term perspective and to provide sustainable and homogeneous coverage of Copernicus Participating countries (EC,2016b). 


\section{Zeil et al}

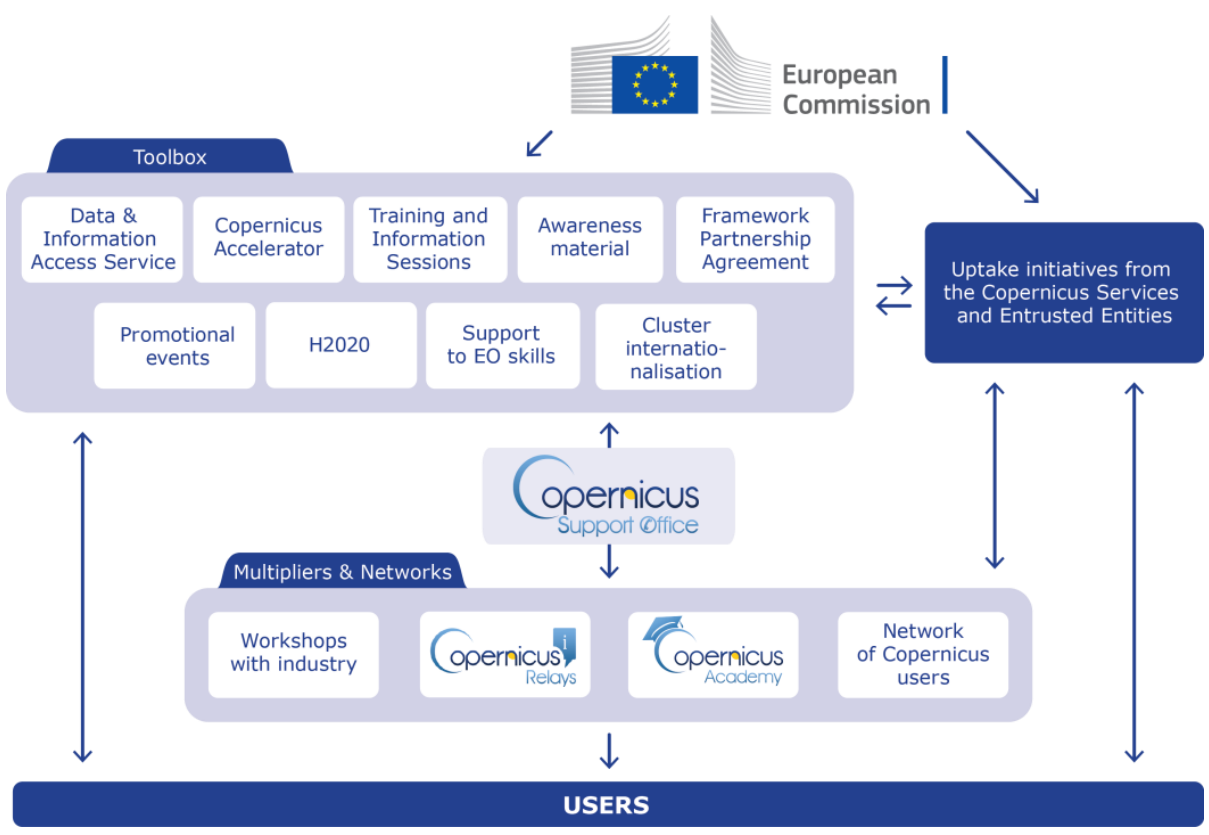

Figure 2: Overall architecture of the user uptake actions (EC,2016b)

In this context, the European Commission has decided to create two dedicated networks:

- the 'Copernicus Relays', a Network of Copernicus helpdesks/information points to engage with national, regional and local stakeholders for Copernicus user uptake

- the 'Copernicus Academy Network' to develop the potential benefits behind the Copernicus data and information products offered

\section{Key elements of the User Uptake strategy}

\section{The Copernicus Support Office}

The Support Office for Copernicus User Uptake facilitates the implementation of the Commission's user uptake initiatives in national and regional action plans, and also ensures cross-fertilization of best practices between the key stakeholders.

Based on the lessons learnt from the study referred to above (EC,2016a), the Copernicus User Uptake Support Office provides support to the Members of the Networks of Copernicus Relays and the Copernicus Academy in order to ensure a comprehensive and integrated approach for user uptake initiatives. It will liaise with Copernicus Participating States via a series of workshops, where comparable countries in terms of user uptake maturity or thematic needs discuss user uptake initiatives.

The Copernicus User Uptake Support Office should be identified by key stakeholders as the permanent one-stop shop and act as the single helpdesk/information point to provide 
stakeholders with technical assistance, and support their efforts in activities that increase awareness. A bottom-up approach is also foreseen, since the structure should also be perceived as a potential user-feedback mechanism for the Commission's services. Increased uptake of Copernicus can be achieved by the Support Office's concrete involvement in broadening the spectrum of multipliers to communities that have no direct connection with space.

The Copernicus User Uptake Support Office will play a key role in the following range of activities:

1. To support Copernicus-relevant actions launched at European, national, regional and local levels, ensuring a broad, coordinated, sustainable and homogeneous coverage in Copernicus Participating States;

2. To help the Commission set up an integrated approach to stimulate uptake in order to maximize the potential of Copernicus in terms of growth and jobs;

3. To help key stakeholders leverage existing tools and resources in order to strengthen the impact of user uptake initiatives;

4. To actively contribute to the running of networks to be set up by the Commission (i.e. Networks of Copernicus Relays and of the Copernicus Academy);

5. To provide support to user uptake initiatives developed by relevant regional and local networks, as well as supporting thematic networks identified by the Commission;

6. To ensure communication and feedback loops.

A one-year renewable contract for the implementation of the Support Office was signed in 2016 with a consortium led by SpaceTecPartners.

\section{Network of Copernicus Relays}

To engage with national, regional and local stakeholders for Copernicus user uptake, a Network of Copernicus contact points called the 'Copernicus Relays' was created. No direct budget is allocated to the members, but the Commission finances the facilitation of the network by the Copernicus Support Office.

The Relays will act as multipliers developing initiatives to reach two different types of objective:

1. To promote Copernicus as a source of free, open and reliable information to meet the needs of local public services.

2. To promote Copernicus as a source of full, free, open and reliable data for the development of environmental services with high commercial potential by local entrepreneurs.

The Relays are committed to organizing and coordinating awareness activities at local and regional levels (i.e. mapping Copernicus-related events, distribution of dissemination materials, creation and running of local hotlines, promoting Copernicus at relevant events, organization of promotional events and training sessions). 


\section{The Copernicus Academy Network}

The mission of the network is to support the Commission in tackling the challenge of bridging the gap between e-skills and data use, and to enable Copernicus data uptake in new sectors. Again, no direct budget is allocated to the members, but the Commission finances the facilitation of the network by the Copernicus Support Office.

Each Academy member will act as a multiplier, developing initiatives to reach different types of objective:

1. Key outcomes of the network could be: the development of interdisciplinary and international Master's and educational classes; new skills-boosting programmes for vocational training; industry-university traineeship agreements; creation of spin-offs; best practices building; the creation of reference tools and materials.

2. As there are numerous applications outside the usual space-related application domains, it is crucial to raise awareness about them within universities and business schools to alert both young and experienced people to the opportunities that the multiple possibilities represent for their professional specialization.

The Commission encourages and aims to support interactions and the sharing of best practices between members of the Network. The Network should be created between members that are willing to become pioneers and ambassadors of the knowledge potential behind Copernicus data and information, and to provide benchmark standards.

The members of the Copernicus Academy Network should benefit from the service provided by the Copernicus Support Office, which would play a key role in the following areas:

- Mapping, monitoring and liaising between Network members with regard to initiatives of interest for the Network, such as events and educational opportunities (summer schools, post-graduate scholarships, etc.) delivered by members of the Network and/or external actors, in order to build up network externalities;

- Maintaining and sharing communication tools to ensure updates about latest events, information sessions, Copernicus lectures, new members, availability of new training tools, best practices, academic curricula, funding opportunities (H2020 calls, ERASMUS+ call for sectorial cooperation on skills, COSME call for earth observation skills etc.);

- Fostering user uptake and Network members' spin-off strategies by interacting with the Copernicus Entrusted Entities, Copernicus partners and local actors, because today's students are tomorrow's entrepreneurs;

- Fostering the memorandum of understanding between the Copernicus Academy Network members by facilitating development of the network;

- Providing tailored lectures and presentations to members;

- Disseminating communications and educational materials and tools.

The Members of the Copernicus Academy Network would play a key role, on a voluntary basis, in: 
- Developing publications and reference lectures for training on Copernicus data and information use, under a public licence;

- Strengthening the synergies and cooperation between members by sharing knowledge-building, best practices and Copernicus-related research and applications outcomes, and by enabling developed innovations to 'hit the market' as quickly as possible;

- Establishing dedicated vocational training and thesis scholarships coordinated in the framework of cooperative arrangements between universities delivering higher educational programmes, or public institutions supporting career training in partnership with universities, and private business actors. The involvement of startups is seen as an asset;

- Building up a knowledge culture and a strategic think-thank around the benefits of the Copernicus programme and its potential for both public services' needs and societal challenges;

- Taking advantage of the synergic actions identified and communicated by the Copernicus Support Office and by the members themselves in order to establish best practices, such as inter-university exchanges of human resources, or clustering EU funding for tailored interdisciplinary education programmes between faculties (e.g. engineering, geography and economics), and/or between universities with the inherent cooperation and participation of industry, associations, incubators, financial institutions and public actors;

- Developing a synergic relationship with the Copernicus Relays Network and other key networks through the coordination of the Commission, in particular with the ESA Education Office and the Knowledge and Innovation Communities (KICs);

- Contributing to the development of the ecosystem that the Commission is building around Copernicus and space data generally, with a focus on the match with market needs and the economic dimension (e.g. the development of spin-offs).

These combined actions should allow the uptake of the existing open and free space data and information products by boosting the development of the professional skills needed by the potential users of space data, where a key added value is given to merging skills and knowledge from different sectors, in particular transversal skills such as IT, GIS and datahandling skills.

\section{Status of implementation}

After the adoption of the European Space Strategy in October 2016 (EC,2016c), an agenda was set in motion with the objective of increasing and strengthening space applications, in particular those based on Copernicus. Recognizing that public authorities have a central role to play by supporting research and innovation, by providing harmonized public services to all EU citizens (notably through the Copernicus services), by driving demand (the public sector still represents $65 \%$ of the demand for EO products), and by establishing appropriate framework conditions for the private sector (offering predictability and planning certainty), Copernicus Relay Members in most of the European Member States were established. As the 
sustainability of the Copernicus programme strongly depends on a vibrant commercial downstream sector, the Copernicus Academy Network is being formed in such as a way as to link authorities with those who can answer their demands, service providers with those who can offer innovative solutions, and re-search organizations with those who, potentially, can apply and develop their research.

Since January 2017, regular teleconferences are organized and moderated by the newly established Copernicus Support Office.

\section{Outlook}

Europe has the second largest public space budget in the world, with programmes and facilities across Europe. Between 2014 and 2020, the EU will invest over EUR 12 billion in space activities. It owns world-class space systems with Copernicus for Earth Observation, and EGNOS and Galileo for satellite navigation and geo-positioning. With 18 satellites currently in orbit and a further 30 planned in the next 10-15 years, the EU is the largest institutional customer for launch services in Europe (EC, 2016c).

Copernicus is one of the leading providers of Earth Observation data. However, technical barriers currently prevent users from fully exploiting the data and information that Copernicus delivers. The Commission therefore aims to improve access to and exploitation of space data, enabling their cross-fertilization with other sources of data, facilitating the integration with digital research infrastructures, in complementarity with the European cloud initiative. These measures will open up new business opportunities for European industry, including SMEs and start-ups, and will allow research institutions, public authorities and companies to develop and benefit from space solutions. This effort should be supported by accompanying measures at national and regional levels, such as the setting up of support networks in the form of the Copernicus Relays and the Copernicus Academy.

\section{References}

EC, 2016a. Copernicus User Uptake- Engaging with public authorities, the private sector and civil society. Final Report. Retrieved from

http://www.copernicus.eu/sites/default/files/library/Copernicus_User_Uptake_Engaging_with_ Users_0.pdf

EC, 2016b. Fostering the Uptake of Copernicus and Space Applications. Retrieved from http://www.copernicus.eu/sites/default/files/documents/User_uptake/Fostering_the_uptake_of _Copernicus_and_Space_applications.pdf

EC, 2016C. Communication from the Commission to the European Parliament, the Council, the European Economic and Social Committee and the Committee of the Regions - Space Strategy for Europe. $\operatorname{COM}(2016) 705$ final. Retrieved from https://ec.europa.eu/transparency/regdoc/rep/1/2016/EN/COM-2016-705-F1-ENMAIN.PDF 